\title{
Heterotic Discrete Flavor Model
}

\author{
Paul H. Frampton ${ }^{1 *}$, Chiu Man $\mathrm{Ho}^{2,3 \dagger}$ and Thomas W. Kephart ${ }^{2 \ddagger}$ \\ ${ }^{1}$ Department of Physics and Astronomy, \\ University of North Carolina, Chapel Hill, NC 27599, USA \\ ${ }^{2}$ Department of Physics and Astronomy, \\ Vanderbilt University, Nashville, TN 37235, USA \\ ${ }^{3}$ Department of Physics and Astronomy, \\ Michigan State University, East Lansing, MI 48824, USA
}

(Dated: August 29, 2018)

\begin{abstract}
We present an extended 331 model with $T^{\prime}$ discrete flavor symmetry that simultaneously explains the need to have exactly three generations and provides acceptable quark and lepton masses and mixings. New fermionic states and gauge bosons are predicted within the reach of the LHC. We discuss the relevance to the $126 \mathrm{GeV}$ scalar discovered at the LHC.
\end{abstract}

PACS numbers: 12.15.Ff, 11.30.Hv, 11.15.Ex, 11.30.Ly

\footnotetext{
* paul.h.frampton@gmail.com

† chiuman.ho@vanderbilt.edu

$\ddagger$ tom.kephart@gmail.com
} 


\section{INTRODUCTION}

The particle at $126 \mathrm{GeV}$ has now been established at the LHC to be consistent with the standard model (SM) Higgs [1, 2], with the correct coupling strengths to fermions [3], CP property [4], and spin [5]. What remains is a definitive extension of the standard model to eliminate our lack of understanding of fermion masses and mixing angles. In addition, while chiral anomaly cancellation restricts the combination of complex irreducible representations (irreps) that fermions can inhabit, but allow SM generations plus right-handed singlet neutrinos, the number of generations is not constrained by anomaly cancellation. Unitarity of the Pontecorvo-Maki-Nakagawa-Sakata (PMNS) [6] and Cabibbo-Kobayashi-Maskawa (CKM) [7] matrices would tell us that the first three generations can be sufficient unto themselves (or at least decoupled from additional fermions if they exist), while lack of unitarity would imply more fermions, either in extensions of the first three generations or in additional generations.

A number of models with discrete symmetries have been proposed to explain the fermion masses and mixings. For recent reviews, see [8] and [9]. One model where many of the masses and mixings are calculable is the $T^{\prime}$ model [10] which has been explored in detail in [11 19], where $T^{\prime}$ is the binary tetrahedral group which is economical in the sense that it has only 24 elements yet still has sufficient irreps $\left(\mathbf{1}_{\mathbf{1}}, \mathbf{1}_{\mathbf{2}}, \mathbf{1}_{\mathbf{3}}, \mathbf{2}_{\mathbf{1}}, \mathbf{2}_{\mathbf{2}}, \mathbf{2}_{\mathbf{3}}\right.$, and $\left.\mathbf{3}\right)$ to arrange masses and mixings in agreement with the experimental data. The top quark is naturally split off from the light quarks by the choice of embedding in $T^{\prime}$, the Cabibbo angle is directly calculable, etc. We tabulate the Kronecker products for the irreducible representations of $T^{\prime}$ [12] in Table I.

The standard model is insufficient for explaining why we have three generations of fermions. Asymptotic freedom does restrict the number, but does not fix it. A simple extended model that does require three generations is the 331 model [20, 21], where the third generation is assigned to the $S U(3)_{C} \times S U(3)_{L} \times U(1)_{X}$ gauge group differently from the first two, and it takes all three generations to cancel the chiral anomalies. (This idea can be extended to a class of models with the gauge group $S U(3)_{C} \times S U(N)_{L} \times U(1)_{X}$ where for all $N \geq 3$, anomaly cancellation requires three generations [22].) This again, as in the $T^{\prime}$ model, allows for a heavy top quark, plus now there are additional quarks and leptons in the 


\begin{tabular}{||c||c|c|c|c|c|c|c||}
\hline \hline & $1_{1}$ & $1_{2}$ & $1_{3}$ & $2_{1}$ & $2_{2}$ & $2_{3}$ & 3 \\
\hline \hline $1_{1}$ & $1_{1}$ & $1_{2}$ & $1_{3}$ & $2_{1}$ & $2_{2}$ & $2_{3}$ & 3 \\
\hline $1_{2}$ & $1_{2}$ & $1_{3}$ & $1_{1}$ & $2_{2}$ & $2_{3}$ & $2_{1}$ & 3 \\
\hline $1_{3}$ & $1_{3}$ & $1_{1}$ & $1_{2}$ & $2_{3}$ & $2_{1}$ & $2_{2}$ & 3 \\
\hline $2_{1}$ & $2_{1}$ & $2_{2}$ & $2_{3}$ & $1_{1}+3$ & $1_{2}+3$ & $1_{3}+3$ & $2_{1}+2_{2}+2_{3}$ \\
\hline $2_{2}$ & $2_{2}$ & $2_{3}$ & $2_{1}$ & $1_{2}+3$ & $1_{3}+3$ & $1_{1}+3$ & $2_{1}+2_{2}+2_{3}$ \\
\hline $2_{3}$ & $2_{3}$ & $2_{1}$ & $2_{2}$ & $1_{3}+3$ & $1_{1}+3$ & $1_{2}+3$ & $2_{1}+2_{2}+2_{3}$ \\
\hline 3 & 3 & 3 & 3 & $2_{1}+2_{2}+2_{3}$ & $2_{1}+2_{2}+2_{3}$ & $2_{1}+2_{2}+2_{3}$ & $1_{1}+1_{2}+1_{3}+3+3$ \\
\hline \hline
\end{tabular}

TABLE I: Kronecker products for the irreducible representations of $T^{\prime}$.

extended generations in addition to the leptoquark gauge bosons due to the extended gauge symmetry. While explaining why there are three generations, the 331 model has limited predictability of the fermion masses and mixings.

We will argue that the extension of the standard model with $T^{\prime}$ discrete flavor symmetry to a $331 \times T^{\prime}$ model avoids some of the short comings of both the $T^{\prime}$ and the 331 models. ${ }^{1}$ Our $331 \times T^{\prime}$ model retains all the predictions of the original $\mathrm{SM} \times T^{\prime}$ model for fermion masses and mixings, while three generations are still dictated by anomaly cancellation as in the 331 model.

In the original 331 model, we need three $S U(3)_{L}$ triplet scalars and one sextet scalar in order to accommodate all the spontaneous symmetry breaking. However, as we will show, the Higgs sector of the $\mathrm{SM} \times T^{\prime}$ model together with only two additional $S U(3)_{L}$ triplet scalars are sufficient for the extension to the $331 \times T^{\prime}$ model. Thus, only a minimal extension of the Higgs sector is required when the $\mathrm{SM} \times T^{\prime}$ model is extended to the $331 \times T^{\prime}$ model and the complicated sextet scalar can be avoided. In general, it is non-trivial that the resulting model is still consistent when two distinct models are merged together; and even if it can be made so, many nontrivial extensions of the spectrum may be required. On the contrary, the extension from $\mathrm{SM} \times T^{\prime}$ to $331 \times T^{\prime}$ retains all the merits of each of 331 and $\mathrm{SM} \times T^{\prime}$, while at the same time, maintaining consistency and simplicity. Besides, many

\footnotetext{
${ }^{1}$ Ref. [23], which has a similar motivation as the current paper, considers $331 \times S_{3}$. While $S_{3}$ is slightly simpler than $T^{\prime}$, it accounts only for quark masses and mixing. However, $T^{\prime}$ accounts for both of quark and lepton masses and mixing.
} 
more predictions of the new model than of either the 331 model or the $\mathrm{SM} \times T^{\prime}$ model are within reach of the LHC. In this sense, the new $331 \times T^{\prime}$ model is more than the sum of its parts and can justifiably be called a "heterotic" model.

Before describing our model, we note that SUSY and fourth-generation models are becoming disfavored by the data, which makes the exploration of alternative extensions of the SM more attractive. (We note that a SUSY $S U(5) \times T^{\prime}$ model has been studied [24] as has a SUSY extended $331 \times T^{\prime}[25]$.)

\section{PARTICLE ASSIGNMENTS}

Let us begin with the particle assignments under the $S U(3)_{C} \times S U(3)_{L} \times U(1)_{X}$ gauge group and $T^{\prime} \times Z_{2}$ discrete symmetry of the $331 \times T^{\prime}$ model. The quantum numbers are written in the following manner:

$$
\left(S U(3)_{C}, S U(3)_{L}, U(1)_{X}, \mathbf{T}^{\prime}, Z_{2}\right)
$$

We embed the left-handed SM leptons in $S U(3)_{L}$ anti-triplets:

$$
\left(\begin{array}{c}
E_{e}^{+} \\
\nu_{e} \\
e^{-}
\end{array}\right)_{L}\left(\begin{array}{c}
E_{\mu}^{+} \\
\nu_{\mu} \\
\mu^{-} \\
E_{\tau}^{+} \\
\nu_{\tau} \\
\tau^{-}
\end{array}\right)_{L} \begin{cases}L_{L}(1, \overline{3}, 0, \mathbf{3},+1) & E_{e R}^{+}\left(1,1,1, \mathbf{1}_{\mathbf{3}},+1\right) \\
& E_{\mu R}^{+}\left(1,1,1, \mathbf{1}_{\mathbf{2}},+1\right) \\
& E_{\tau R}^{+}\left(1,1,1, \mathbf{1}_{\mathbf{1}},+1\right) \\
& \\
\end{cases}
$$

where there is an additional charged heavy lepton per triplet whose right-handed partners are in singlets as are the right-handed partners of the SM charged leptons. Three righthanded neutrinos are included for use in the see-saw mechanism as needed for masses and mixings:

$$
\begin{array}{ll}
e_{R}^{-}\left(1,1,-1, \mathbf{1}_{\mathbf{3}},-1\right) & N_{R}^{(1)}\left(1,1,0, \mathbf{1}_{\mathbf{1}},+1\right) \\
\mu_{R}^{-}\left(1,1,-1, \mathbf{1}_{\mathbf{2}},-1\right) & N_{R}^{(2)}\left(1,1,0, \mathbf{1}_{\mathbf{2}},+1\right) \\
\tau_{R}^{-}\left(1,1,-1, \mathbf{1}_{\mathbf{1}},-1\right) & N_{R}^{(3)}\left(1,1,0, \mathbf{1}_{\mathbf{3}},+1\right) .
\end{array}
$$


The 331 charges and irreps are dictated by anomaly cancellation (except for the right-handed neutrinos). The discrete group assignments are similar to and extend those of the $\mathrm{SM} \times T^{\prime}$ model.

The SM quarks are also assigned as in the $\mathrm{SM} \times T^{\prime}$ model with extended generation quarks included naturally as we increase the $S U(2)_{L}$ gauge symmetry to $S U(3)_{L}$ :

$$
\left.\begin{array}{l}
\left(\begin{array}{l}
b \\
t \\
T
\end{array}\right)^{L} \mathcal{Q}_{L} \quad\left(3, \overline{3}, \frac{2}{3}, \mathbf{1}_{\mathbf{1}},+1\right) \\
\left(\begin{array}{l}
c \\
s \\
S
\end{array}\right)_{L} \\
u \\
d \\
D
\end{array}\right)_{L} Q_{L} \quad\left(3,3,-\frac{1}{3}, \mathbf{2}_{\mathbf{1}},+1\right)
$$

Using the convention $\operatorname{Tr}\left(\lambda_{\mathrm{a}} \lambda_{\mathrm{b}}\right)=2 \delta_{\mathrm{ab}}$, two of the generators for $S U(3)_{L}$ are given by $\lambda_{3}=\operatorname{diag}(1,-1,0)$ and $\lambda_{8}=\frac{1}{\sqrt{3}} \operatorname{diag}(1,1,-2)$. After the symmetry $S U(3)_{L} \times U(1)_{X}$ breaks down to $S U(2)_{L} \times U(1)_{Y}$, the hypercharge $Y$ is [20]:

$$
Y=2 X+\sqrt{3} \lambda_{8}
$$

and the electric charge is given by $Q=T_{3}+\frac{Y}{2}$ where $T_{3}=\frac{1}{2} \lambda_{3}$. The right-handed SM quarks are again as in the $\mathrm{SM} \times T^{\prime}$ model, but the new heavy right-handed quarks are arranged with $T_{R}$ in a $T^{\prime}$ singlet and $S_{R}$ and $D_{R}$ in a $T^{\prime}$ doublet:

$$
\begin{aligned}
& T_{R} \quad\left(3,1, \frac{5}{3}, \mathbf{1}_{\mathbf{1}},+1\right) \\
& \left.\begin{array}{l}
S_{R} \\
D_{R}
\end{array}\right\} \mathcal{D}_{R} \quad\left(3,1,-\frac{4}{3}, \mathbf{2}_{\mathbf{1}},+1\right) \\
& t_{R} \quad\left(3,1, \frac{2}{3}, \mathbf{1}_{\mathbf{1}},+1\right) \\
& b_{R} \quad\left(3,1,-\frac{1}{3}, \mathbf{1}_{\mathbf{2}},-1\right) \\
& \left.c_{u_{R}}\right\} \mathcal{C}_{R} \quad\left(3,1, \frac{2}{3}, \mathbf{2}_{\mathbf{3}},-1\right) \\
& \left.\begin{array}{l}
s_{R} \\
d_{R}
\end{array}\right\} \mathcal{S}_{R} \quad\left(3,1,-\frac{1}{3}, \mathbf{2}_{\mathbf{2}},+1\right) \text {. }
\end{aligned}
$$


We only need to extend the Higgs sector of the $\mathrm{SM} \times T^{\prime}$ model by two $S U(3)_{L}$ triplet scalars in order to accommodate the additional spontaneous symmetry breaking needed in the 331 sector. Hence, we choose the Higgses $H_{\mathbf{3}}, H_{\mathbf{3}}^{\prime}, H_{\mathbf{1}_{\mathbf{1}}}, H_{\mathbf{1}_{\mathbf{3}}}$ as in [13] together with the two extra scalars $\Phi_{3}$ and $\Phi_{1_{1}}$ :

$$
\begin{aligned}
& H_{\mathbf{3}}(1, \overline{3}, 0, \mathbf{3},+1) \\
& H_{\mathbf{3}}^{\prime}(1, \overline{3}, 1, \mathbf{3},-1) \\
& H_{\mathbf{1}_{\mathbf{1}}}\left(1, \overline{3}, 0, \mathbf{1}_{\mathbf{1}},+1\right) \\
& H_{\mathbf{1}_{\mathbf{3}}}\left(1, \overline{3}, 1, \mathbf{1}_{\mathbf{3}},-1\right) \\
& \Phi_{\mathbf{3}}(1,3,1, \mathbf{3},+1) \\
& \Phi_{\mathbf{1}_{\mathbf{1}}}\left(1,3,1, \mathbf{1}_{\mathbf{1}},+1\right) .
\end{aligned}
$$

These six scalars can acquire their respective vacuum expectation values (VEVs) through the Higgs potential shown in Appendix A.

At this point, we can write down the most general Yukawa lagrangian for the lepton sector

$$
\begin{aligned}
& \mathcal{L}_{Y}^{\text {leptons }}=\frac{1}{2} M_{1} \bar{N}_{R}^{(1)} N_{R}^{(1)}+M_{23} \bar{N}_{R}^{(2)} N_{R}^{(3)} \\
& +\left\{Y_{1}\left(\bar{L}_{L} N_{R}^{(1)} H_{3}\right)+Y_{2}\left(\bar{L}_{L} N_{R}^{(2)} H_{3}\right)+Y_{3}\left(\bar{L}_{L} N_{R}^{(3)} H_{3}\right)\right. \\
& +Y_{e}\left(\bar{L}_{L} e_{R} H_{3}^{\prime}\right)+Y_{\mu}\left(\bar{L}_{L} \mu_{R} H_{3}^{\prime}\right)+Y_{\tau}\left(\bar{L}_{L} \tau_{R} H_{3}^{\prime}\right) \\
& \left.+Y_{e}^{\prime}\left(\bar{L}_{L} E_{e R} \Phi_{3}^{*}\right)+Y_{\mu}^{\prime}\left(\bar{L}_{L} E_{\mu R} \Phi_{3}^{*}\right)+Y_{\tau}^{\prime}\left(\bar{L}_{L} E_{\tau R} \Phi_{3}^{*}\right)\right\}+ \text { h.c. }
\end{aligned}
$$

and for the quark sector

$$
\begin{aligned}
\mathcal{L}_{Y}^{\text {quarks }}= & Y_{t}\left(\left\{\overline{\mathcal{Q}}_{L}\right\}_{\mathbf{1}_{\mathbf{1}}}\left\{t_{R}\right\}_{\mathbf{1}_{\mathbf{1}}} H_{\mathbf{1}_{\mathbf{1}}}\right)+Y_{b}\left(\left\{\overline{\mathcal{Q}}_{L}\right\}_{\mathbf{1}_{\mathbf{1}}}\left\{b_{R}\right\}_{\mathbf{1}_{\mathbf{2}}} H_{\mathbf{1}_{\mathbf{3}}}\right) \\
& +Y_{\mathcal{C}}\left(\left\{\bar{Q}_{L}\right\}_{\mathbf{2}_{\mathbf{1}}}\left\{\mathcal{C}_{R}\right\}_{\mathbf{2}_{\mathbf{3}}} H_{\mathbf{3}}^{*}\right)+Y_{\mathcal{S}}\left(\left\{\bar{Q}_{L}\right\}_{\mathbf{2}_{\mathbf{1}}}\left\{\mathcal{S}_{R}\right\}_{\mathbf{2}_{\mathbf{2}}} H_{\mathbf{3}}^{*}\right) \\
& +Y_{T}\left(\left\{\overline{\mathcal{Q}}_{L}\right\}_{\mathbf{1}_{\mathbf{1}}}\left\{T_{R}\right\}_{\mathbf{1}_{\mathbf{1}}} \Phi_{\mathbf{1}_{\mathbf{1}}}^{*}\right)+Y_{\mathcal{D}}\left(\left\{\bar{Q}_{L}\right\}_{\mathbf{2}_{\mathbf{1}}}\left\{\mathcal{D}_{R}\right\}_{\mathbf{2}_{\mathbf{1}}} \Phi_{\mathbf{1}_{\mathbf{1}}}\right) \\
& + \text { h.c. }
\end{aligned}
$$

In this $331 \times T^{\prime}$ model, the neutrino sector is unchanged relative to the $\mathrm{SM} \times T^{\prime}$ model. So the predictions for the masses and mixings of the neutrino sector in the $331 \times T^{\prime}$ model are exactly those predicted by the $\mathrm{SM} \times T^{\prime}$ model. The $\mathrm{SM}$ quark and charged-lepton 
Yukawa terms are unchanged as well. The masses of the charged heavy leptons $E_{e}^{ \pm}, E_{\mu}^{ \pm}, E_{\tau}^{ \pm}$ are determined by the product of $Y^{\prime}$ and $\left\langle\Phi_{3}\right\rangle$, which can be chosen at the TeV scale. Furthermore, the masses of the heavy quarks $T, S, D$ inherited from the 331 model could also be produced at the TeV scale through the appropriate choices of new Yukawa couplings and $\left\langle\Phi_{\mathbf{1}_{\mathbf{1}}}\right\rangle$. For instance, if we assume that the new Yukawa couplings are of order unity, then we simply require $\left\langle\Phi_{\mathbf{1}_{1}}\right\rangle$ to be $\mathrm{TeV}$ scale.

\section{ANOMALIES}

There are six types of triangle anomalies. Namely, $S U(3)_{C}^{3}, \quad S U(3)_{C}^{2} X, \quad S U(3)_{L}^{3}$, $S U(3)_{L}^{2} X, X^{3}$ and $X$, where the last anomaly is from a mixed gauge-gravity triangle diagram. In the $331 \times T^{\prime}$ model, the left-handed leptons are assigned in $S U(3)_{L}$ anti-triplets with the same $S U(3)_{C} \times S U(3)_{L} \times U(1)_{X}$ quantum numbers as those in the original 331 model. The first two and the third generations of quarks also carry exactly the same quantum numbers as those in the original 331 model. The right-handed neutrinos are irrelevant for any anomaly cancellations as they are singlets of $S U(3)_{C} \times S U(3)_{L} \times U(1)_{X}$. Since the right-handed charged leptons are singlets under $S U(3)_{C} \times S U(3)_{L}$, they do not participate in the $S U(3)_{C}^{3}, S U(3)_{C}^{2} X, S U(3)_{L}^{3}, S U(3)_{L}^{2} X$ anomaly cancellations. Thus, cancellations of these anomalies proceed in the same way as in the original 331 model, which require three generations. For the $X^{3}$ and $X$ anomalies, the contributions from $e_{R}, \mu_{R}, \tau_{R}$ cancel those from $E_{e R}, E_{\mu R}, E_{\tau R}$ respectively and this happens generation by generation. However, for the rest of the $X^{3}$ and $X$ anomalies, cancellations proceed in the same way as in the original 331 model, which again require three generations.

Therefore, our new $331 \times T^{\prime}$ model retains all the acceptable predictions for fermion masses and mixings contained in the original $321 \times T^{\prime}$ model, while three generations are still required by anomaly cancellation as in the original 331 model.

It is possible that the discrete symmetry $T^{\prime}$ originates from a discrete gauge symmetry that is spontaneously broken. The advantage of having a gauge origin is that $T^{\prime}$ could then be protected against violations by quantum gravity effects [26]. But the introduction of a new discrete gauge symmetry may also imply the possibility of discrete gauge anomalies [27, 28]. The requirement of discrete gauge anomaly cancellations leads to the discrete anomaly conditions after the discrete gauge symmetry is broken. It is remarkable that our 
model with $T^{\prime}$ symmetry is discrete anomaly free (see it e.g. the second row of Table 2 in [29]).

\section{YUKAWA COUPLINGS TO 126 GEV SCALAR}

The discovery of a resonance at $\sim 126 \mathrm{GeV}$ at the LHC [1, 2], strongly suggestive of the Higgs boson, has naturally caused intense interest. Its preliminary properties are consistent within errors with the Higgs particle predicted by the minimal standard model. The twobody decays which can be measured accurately in the near future include $H \rightarrow \gamma \gamma, H \rightarrow \bar{b} b$, and $H \rightarrow \bar{\tau} \tau$. These are respectively sensitive to the Yukawa couplings $Y_{H \bar{t} t}$ (through the top triangle contribution which competes with the $W$-loop), $Y_{H \bar{b} b}$, and $Y_{H \bar{\tau} \tau}$.

In the minimal standard model, the Yukawa couplings $Y_{H \bar{f} f}$ appear in the simple form $Y_{H \bar{f} f} \bar{f} f H$ so that they are proportional to the masses

$$
Y_{H \bar{f} f} \propto m_{f},
$$

where the proportionality constant is $1 / v$ with $v$ being the vacuum expectation value of the Higgs field. In the current renormalizable model with a non-trivial flavor symmetry $G_{F}=T^{\prime} \times Z_{2}$, there are several Higgs and the Yukawa couplings of the lightest one will generally deviate from the simple proportionality of Eq. (10). Such deviations may likely be small but crucial to understanding how the group $G_{F}$ operates. One may even say that if the conventional prediction of Eq. (10) would hold exactly at high precision, then the renormalizable $G_{F}$ models would be disfavored.

The statements above are true for general renormalizable $G_{F}$ models. To illustrate them, we focus on the present choice $G_{F}=T^{\prime} \times Z_{2}$ [10] and the minimal model as previously discussed in [13]. We shall concentrate only on the third-generation couplings $Y_{H \bar{f} f}$ for $f=t, b, \tau$. Imposing strict renormalizability on the lepton lagrangian allows nontrivial terms for the $\tau$ mass:

$$
Y_{\tau}\left(\bar{L}_{L} \tau_{R} H_{3}^{\prime}\right)+\text { h.c. }
$$

where $H_{\mathbf{3}}^{\prime}$ transforms as $H_{\mathbf{3}}^{\prime}(1, \overline{3}, 1, \mathbf{3},-1)$. The Yukawa couplings to the third-generation of quarks are contained in

$$
\mathcal{L}_{3 \mathrm{rd}}^{\text {quarks }}=Y_{t}\left(\left\{\overline{\mathcal{Q}}_{L}\right\}_{\mathbf{1}_{\mathbf{1}}}\left\{t_{R}\right\}_{\mathbf{1}_{\mathbf{1}}} H_{\mathbf{1}_{\mathbf{1}}}\right)+Y_{b}\left(\left\{\overline{\mathcal{Q}}_{L}\right\}_{\mathbf{1}_{\mathbf{1}}}\left\{b_{R}\right\}_{\mathbf{1}_{\mathbf{2}}} H_{\mathbf{1}_{\mathbf{3}}}\right)+\text { h.c. }
$$


No $T^{\prime}$ doublet $\left(\mathbf{2}_{\mathbf{1}}, \mathbf{2}_{\mathbf{2}}, \mathbf{2}_{\mathbf{3}}\right)$ scalars have been added. This allows a non-zero value only for $\Theta_{12}$. The other CKM angles vanish, making the third generation stable and $m_{b}>m_{s, d}$ as outlined in [10]. Such a model leads to the formula [13] for the Cabibbo angle

$$
\tan 2 \Theta_{12}=\left(\frac{\sqrt{2}}{3}\right),
$$

or equivalently, $\sin \Theta_{12}=0.218$.., which is close to the experimental value $\sin \Theta_{12} \simeq 0.227$. It can also lead to the successful relationship between neutrino mixing angles $\theta_{i j}$

$$
\theta_{13}=(\sqrt{2})^{-1}\left|\frac{\pi}{4}-\theta_{23}\right|,
$$

which is in excellent agreement with the latest experiments [30].

In such a model, the lightest Higgs $H$ is a linear combination of other Higgs:

$$
H=a H_{1_{1}}+b H_{1_{3}}+c H_{3}^{\prime}+\ldots,
$$

and the consequent Yukawa couplings are

$$
Y_{H \bar{t} t}=a^{-1} Y_{t}, \quad Y_{H \bar{b} b}=b^{-1} Y_{b}, \quad Y_{H \bar{\tau} \tau}=c^{-1} Y_{\tau} .
$$

The VEV $v$ is shared between the $\left\langle H_{\alpha}>\left(\alpha=\mathbf{1}_{\mathbf{1}}, \mathbf{1}_{\mathbf{3}}, \mathbf{3}, \ldots\right)\right.$ irreps of $T^{\prime}$ and there is no reason to expect $a=b=c=\ldots$ so that the proportionality of Eq. (10) will generally be lost. In fact, if Eq. (10) remained exact, the only solution would be a trivial one where all states transform as $\mathbf{1}_{\mathbf{1}}$ of $T^{\prime}$ and the $G_{F}$ is inapplicable. The successes in [13] and [30] would, in such a case, be accidental. On the other hand, if Eq. (10) is inexact, the evaluations of the coefficients $a, b, c, \ldots$ can then be used to understand more perspicuously the derivations of mixing angles for quarks and leptons given respectively in [13] and [30], in a first clear departure from the minimal standard model.

\section{DISCUSSION}

One of the perpetual difficulties one encounters in constructing models of fermionic flavor is the necessity of an extended Higgs sector. Typically, if more symmetry is added, then more scalars are needed to break that symmetry and generate the wanted structure of masses and mixings. The model at hand is no exception, although it is somewhat more attractive than average since all the scalars are in fundamental irreps of the $S U(3)_{L}$ in the 
electroweak gauge group. (This is an improvement on previous 331 models that required a 6 scalar of $S U(3)_{L}$.) This simplification leads to fewer phenomenological problem, e.g., the $\rho$ parameter is unchanged ent heterotic model provides new candidates for particles to be discovered in the $\mathrm{TeV}$ range by, for example, the LHC. As new gauge bosons, there are the bileptons familiar from the 331 model which come both doubly-charged and singly-charged in the $S U(2)$ doublets $\left(Y^{++}, Y^{+}\right)$and $\left(Y^{--}, Y^{-}\right)$with striking signatures in like-sign lepton pairs; also there is a $Z^{\prime}$. From muonium-antimuonium conversion experiments [31], a lower bound on the bilepton mass $M_{Y}>850 \mathrm{GeV}$ has been deduced, although this assumed flavor diagonality of the bilepton couplings. More general analysis of bilepton production at the LHC, with weaker assumptions, appears in [32] and [33].

There are additional fermions beyond the standard model. These include the exotic quarks $D$ and $S$ with charge $Q=-4 / 3$ and the $T$ with $Q=+5 / 3$. There are also three new charged leptons, one per family, $E_{e}, E_{\mu}$, and $E_{\tau}$. All of these additional states are predicted to be in the $\mathrm{TeV}$ or multi-TeV range, accessible to the LHC especially at its full energy and luminosity.

\section{Acknowledgments}

The work of P.H.F. was supported in part by U.S. Department of Energy Grant No. DEFG02-05ER41418. C.M.H. and T.W.K. were supported in part by U.S. Department of Energy Grant No. DE-FG05-85ER40226. 


\section{Appendix A: Higgs Potential}

The VEVs for $H_{3}, H_{3}^{\prime}, H_{1_{1}}, H_{1_{3}}, \Phi_{3}$ and $\Phi_{1_{1}}$ can be obtained from minimizing the following Higgs potential:

$$
\begin{aligned}
& V=\lambda_{1}\left(H_{\mathbf{3}}^{\dagger} H_{\mathbf{3}}-v_{\mathbf{3}}^{2}\right)^{2}+\lambda_{2}\left(H_{\mathbf{3}}^{\prime \dagger} H_{\mathbf{3}}^{\prime}-v_{\mathbf{3}}^{\prime 2}\right)^{2}+\lambda_{3}\left(H_{\mathbf{1}_{\mathbf{1}}}^{\dagger} H_{\mathbf{1}_{\mathbf{1}}}-v_{\mathbf{1}_{\mathbf{1}}}^{2}\right)^{2} \\
& +\lambda_{4}\left(H_{\mathbf{1}_{3}}^{\dagger} H_{\mathbf{1}_{3}}-v_{\mathbf{1}_{3}}^{2}\right)^{2}+\lambda_{5}\left(\Phi_{\mathbf{3}}^{\dagger} \Phi_{\mathbf{3}}-v_{\mathbf{3}}^{\prime \prime 2}\right)^{2}+\lambda_{6}\left(\Phi_{\mathbf{1}_{1}}^{\dagger} \Phi_{\mathbf{1}_{1}}-v_{\mathbf{1}_{1}}^{\prime 2}\right)^{2} \\
& +\lambda_{7}\left[\left(H_{\mathbf{3}}^{\dagger} H_{\mathbf{3}}-v_{\mathbf{3}}^{2}\right)+\left(H_{\mathbf{3}}^{\prime \dagger} H_{\mathbf{3}}^{\prime}-v_{\mathbf{3}}^{\prime 2}\right)\right]^{2}+\lambda_{8}\left[\left(H_{\mathbf{3}}^{\dagger} H_{\mathbf{3}}-v_{\mathbf{3}}^{2}\right)+\left(H_{\mathbf{1}_{1}}^{\dagger} H_{\mathbf{1}_{\mathbf{1}}}-v_{\mathbf{1}_{\mathbf{1}}}^{2}\right)\right]^{2} \\
& +\lambda_{9}\left[\left(H_{\mathbf{3}}^{\dagger} H_{\mathbf{3}}-v_{\mathbf{3}}^{2}\right)+\left(H_{\mathbf{1}_{3}}^{\dagger} H_{\mathbf{1}_{3}}-v_{\mathbf{1}_{3}}^{2}\right)\right]^{2}+\lambda_{10}\left[\left(H_{\mathbf{3}}^{\dagger} H_{\mathbf{3}}-v_{\mathbf{3}}^{2}\right)+\left(\Phi_{\mathbf{3}}^{\dagger} \Phi_{\mathbf{3}}-v_{\mathbf{3}}^{\prime \prime 2}\right)\right]^{2} \\
& +\lambda_{11}\left[\left(H_{\mathbf{3}}^{\dagger} H_{3}-v_{\mathbf{3}}^{2}\right)+\left(\Phi_{\mathbf{1}_{1}}^{\dagger} \Phi_{\mathbf{1}_{1}}-v_{\mathbf{1}_{1}}^{\prime 2}\right)\right]^{2}+\lambda_{12}\left[\left(H_{\mathbf{3}}^{\prime \dagger} H_{\mathbf{3}}^{\prime}-v_{\mathbf{3}}^{\prime 2}\right)+\left(H_{\mathbf{1}_{1}}^{\dagger} H_{1_{1}}-v_{\mathbf{1}_{1}}^{2}\right)\right]^{2} \\
& +\lambda_{13}\left[\left(H_{\mathbf{3}}^{\prime \dagger} H_{\mathbf{3}}^{\prime}-v_{\mathbf{3}}^{\prime 2}\right)+\left(H_{\mathbf{1}_{\mathbf{3}}}^{\dagger} H_{\mathbf{1}_{3}}-v_{\mathbf{1}_{3}}^{2}\right)\right]^{2}+\lambda_{14}\left[\left(H_{\mathbf{3}}^{\prime \dagger} H_{\mathbf{3}}^{\prime}-v_{\mathbf{3}}^{\prime 2}\right)+\left(\Phi_{\mathbf{3}}^{\dagger} \Phi_{\mathbf{3}}-v_{\mathbf{3}}^{\prime \prime 2}\right)\right]^{2} \\
& +\lambda_{15}\left[\left(H_{3}^{\prime \dagger} H_{3}^{\prime}-v_{3}^{\prime 2}\right)+\left(\Phi_{1_{1}}^{\dagger} \Phi_{1_{1}}-v_{\mathbf{1}_{1}}^{\prime 2}\right)\right]^{2}+\lambda_{16}\left[\left(H_{\mathbf{1}_{1}}^{\dagger} H_{\mathbf{1}_{1}}-v_{\mathbf{1}_{1}}^{2}\right)+\left(H_{\mathbf{1}_{3}}^{\dagger} H_{\mathbf{1}_{3}}-v_{\mathbf{1}_{3}}^{2}\right)\right]^{2} \\
& +\lambda_{17}\left[\left(H_{\mathbf{1}_{1}}^{\dagger} H_{\mathbf{1}_{1}}-v_{\mathbf{1}_{1}}^{2}\right)+\left(\Phi_{\mathbf{3}}^{\dagger} \Phi_{\mathbf{3}}-v_{\mathbf{3}}^{\prime \prime 2}\right)\right]^{2}+\lambda_{18}\left[\left(H_{\mathbf{1}_{1}}^{\dagger} H_{\mathbf{1}_{1}}-v_{\mathbf{1}_{1}}^{2}\right)+\left(\Phi_{\mathbf{1}_{1}}^{\dagger} \Phi_{\mathbf{1}_{1}}-v_{\mathbf{1}_{1}}^{\prime 2}\right)\right]^{2} \\
& +\lambda_{19}\left[\left(H_{\mathbf{1}_{3}}^{\dagger} H_{\mathbf{1}_{3}}-v_{\mathbf{1}_{3}}^{2}\right)+\left(\Phi_{\mathbf{3}}^{\dagger} \Phi_{\mathbf{3}}-v_{\mathbf{3}}^{\prime \prime 2}\right)\right]^{2}+\lambda_{20}\left[\left(H_{\mathbf{1}_{3}}^{\dagger} H_{\mathbf{1}_{3}}-v_{\mathbf{1}_{3}}^{2}\right)+\left(\Phi_{\mathbf{1}_{\mathbf{1}}}^{\dagger} \Phi_{\mathbf{1}_{1}}-v_{\mathbf{1}_{1}}^{\prime 2}\right)\right]^{2} \\
& +\lambda_{21}\left[\left(\Phi_{\mathbf{3}}^{\dagger} \Phi_{\mathbf{3}}-v_{\mathbf{3}}^{\prime \prime 2}\right)+\left(\Phi_{\mathbf{1}_{1}}^{\dagger} \Phi_{\mathbf{1}_{1}}-v_{\mathbf{1}_{1}}^{\prime 2}\right)\right]^{2} \\
& +\alpha_{1}\left(H_{3} H_{3} H_{3}+\text { h.c. }\right)+\alpha_{2}\left(H_{3} H_{3} H_{1_{1}}+\text { h.c. }\right)+\alpha_{3}\left(H_{1_{1}} H_{1_{1}} H_{1_{1}}+\text { h.c. }\right) \text {, }
\end{aligned}
$$

where all the coefficients $\lambda_{1}, \ldots, \lambda_{21}$ are non-negative. Besides, $v_{\mathbf{3}}, v_{\mathbf{3}}^{\prime}, v_{\mathbf{1}_{\mathbf{1}}}, v_{\mathbf{1}_{\mathbf{3}}}, v_{\mathbf{3}}^{\prime \prime}$ and $v_{\mathbf{1}_{1}}^{\prime}$ are the would-be VEVs for $H_{3}, H_{3}^{\prime}, H_{1_{1}}, H_{1_{3}}, \Phi_{3}$ and $\Phi_{1_{1}}$ respectively. Since the triplets of $T^{\prime}$ are self-conjugate, any cubic combinations of $\mathbf{3}$ are $T^{\prime}$ invariant. The $S U(3)_{L}$ cubic invariant is either $3 \times 3 \times 3$ or $\overline{3} \times \overline{3} \times \overline{3}$. Originally, there are 56 possible Higgs cubic terms. However, 23 of them violate the $Z_{2}$ symmetry, 21 of them are either not invariant under $S U(3)_{L}$ or $U(1)_{X}$, and 9 of them are not invariant under $T^{\prime}$. So we are left with only 3 cubic terms. As long as $\alpha_{1}, \alpha_{2}, \alpha_{3}$ are sufficiently less than $\lambda_{1}, \ldots, \lambda_{21}$, the cubic terms can be treated as small perturbations and do not alter the pattern of symmetry breaking. The true VEVs should be approximately $v_{\mathbf{3}}, v_{\mathbf{3}}^{\prime}, v_{\mathbf{1}_{\mathbf{1}}}, v_{\mathbf{1}_{\mathbf{3}}}, v_{\mathbf{3}}^{\prime \prime}$ and $v_{\mathbf{1}_{\mathbf{1}}}^{\prime}$. Since $\lambda_{1}, \ldots, \lambda_{21}$ are non-negative, these VEVs minimize the Higgs potential. We plan to return to the issue of 
perturbations involving these cubic terms in the near future.

[1] G. Aad et al. [ATLAS Collaboration], Phys. Lett. B 716, 1 (2012) arXiv:1207.7214 [hep-ex].

[2] S. Chatrchyan et al. [CMS Collaboration], Phys. Lett. B 716, 30 (2012) arXiv:1207.7235 [hep-ex].

[3] ATLAS Collaboration, ATLAS-CONF-2013-034; CMS Collaboration, CMS PAS HIG-13-005.

[4] S. Chatrchyan et al. [CMS Collaboration], Phys. Rev. Lett. 110, 081803 (2013) arXiv:1212.6639 [hep-ex]. ATLAS Collaboration, ATLAS-CONF-2013-013; CMS Collaboration, CMS PAS HIG-13-002.

[5] ATLAS Collaboration, ATLAS-CONF-2013-031; CMS Collaboration, CMS PAS HIG-13-003; ATLAS Collaboration, ATLAS-CONF-2013-029 and ATLAS-CONF-2013-040.

[6] B. Pontecorvo, Sov. Phys. JETP 6, 429 (1957) [Zh. Eksp. Teor. Fiz. 33, 549 (1957)]; B. Pontecorvo, Sov. Phys. JETP 26, 984 (1968) [Zh. Eksp. Teor. Fiz. 53, 1717 (1967)]; Z. Maki, M. Nakagawa and S. Sakata, Prog. Theor. Phys. 28, 870 (1962).

[7] N. Cabibbo, Phys. Rev. Lett. 10, 531 (1963); M. Kobayashi and T. Maskawa, Prog. Theor. Phys. 49, 652 (1973).

[8] G. Altarelli and F. Feruglio, Rev. Mod. Phys. 82, 2701 (2010) arXiv:1002.0211 [hep-ph].

[9] H. Ishimori, T. Kobayashi, H. Ohki, Y. Shimizu, H. Okada and M. Tanimoto, Prog. Theor. Phys. Suppl. 183, 1 (2010) arXiv:1003.3552 [hep-th].

[10] P. H. Frampton and T. W. Kephart, Int. J. Mod. Phys. A 10, 4689 (1995) hep-ph/9409330.

[11] A. Aranda, C. D. Carone and R. F. Lebed, Phys. Rev. D 62, 016009 (2000) hep-ph/0002044.

[12] P. H. Frampton and T. W. Kephart, JHEP 0709, 110 (2007) arXiv:0706.1186 [hep-ph].

[13] P. H. Frampton, T. W. Kephart and S. Matsuzaki, Phys. Rev. D 78, 073004 (2008) arXiv:0807.4713 [hep-ph].

[14] D. A. Eby, P. H. Frampton and S. Matsuzaki, Phys. Lett. B 671, 386 (2009) arXiv:0810.4899 [hep-ph].

[15] D. A. Eby, P. H. Frampton and S. Matsuzaki, Phys. Rev. D 80, 053007 (2009) arXiv:0907.3425 [hep-ph].

[16] C. M. Ho and T. W. Kephart, Phys. Lett. B 687, 201 (2010) arXiv:1001.3696 [hep-ph]. 
[17] P. H. Frampton, C. M. Ho, T. W. Kephart and S. Matsuzaki, Phys. Rev. D 82, 113007 (2010) arXiv:1009.0307 [hep-ph].

[18] D. A. Eby, P. H. Frampton, X. -G. He and T. W. Kephart, Phys. Rev. D 84, 037302 (2011) arXiv:1103.5737 [hep-ph]

[19] For a recent review of the $\mathrm{SM} \times T^{\prime}$ model see D.A. Eby, Binary Tetrahedral Flavor Symmetry, Ph D thesis, UNC Chapel Hill, April 2013.

[20] P. H. Frampton, Phys. Rev. Lett. 69, 2889 (1992).

[21] F. Pisano and V. Pleitez, Phys. Rev. D 46, 410 (1992) hep-ph/9206242.

[22] P. H. Frampton, C. M. Ho and T. W. Kephart, Phys. Lett. B 715, 275 (2012) arXiv:1205.4483 [hep-ph].

[23] A. E. Crcamo Hernández, R. Martínez and F. Ochoa, arXiv:1309.6567 [hep-ph].

[24] M. -C. Chen and K. T. Mahanthappa, Phys. Lett. B 652, 34 (2007) arXiv:0705.0714 [hep-ph].

[25] S. Sen, Phys. Rev. D 76, 115020 (2007) arXiv:0710.2734 [hep-ph].

[26] L. M. Krauss and F. Wilczek, Phys. Rev. Lett. 62, 1221 (1989).

[27] L. E. Ibanez and G. G. Ross, Phys. Lett. B 260, 291 (1991).

[28] C. Luhn and P. Ramond, JHEP 0807, 085 (2008) arXiv:0805.1736 [hep-ph].

[29] C. Luhn, Phys. Lett. B 670, 390 (2009) arXiv:0807.1749 [hep-ph].

[30] D.A.Eby and P.H. Frampton, Phys. Rev. D86, 117304 (2012). arXiv:1112.2675[hep-ph]

[31] L. Willmann, et al. Phys. Rev. Lett. 82, 49 (1999).

[32] B. Meirose and A. A. Nepomucheno, Phys. Rev. D84, 055002 (2011) arXiv:1105.6299 [hep-ph].

[33] J. Sa Borges, Y. A. Coutinho, and E.A. Barreto, AIP Conf. Proc. 1520, 440 (2012). 